\section{On the discovery of new elements (IUPAC/IUPAP Report)}

Hofmann, S., Dmitriev, S. N., Fahlander, C., Gates, J.

M., Roberto, J. B., and Sakai, H.

Pure and Applied Chemistry, 2020

published online ahead of print 4 Aug 2020

https://doi.org/10.1515/pac-2020-2926

Almost thirty years ago the criteria that are currently used to verify claims for the discovery of a new element were set down by the comprehensive work of a Transfermium Working Group, TWG, jointly established by IUPAC and IUPAP. The recent completion of the naming of the 118 elements in the first seven periods of the Periodic Table of the Elements was considered as an opportunity for a review of these criteria in the light of the experimental and theoretical advances in the field. In late 2016 the Unions decided to establish a new Joint Working Group, JWG, consisting of six members determined by the Unions. A first meeting of the JWG was in May 2017. One year later this report was finished. In a first part the works and conclusions of the TWG and the Joint Working Parties, JWP, deciding on the discovery of the now named elements are summarized. Possible experimental developments for production and identification of new elements beyond the presently known ones are estimated. Criteria and guidelines for establishing priority of discovery of these potential new elements are presented. Special emphasis is given to a description for the application of the criteria and the limits for their applicability.

\section{A concise guide to polymer nomencla- ture for authors of papers and reports in polymer science and technology (IUPAC Technical Report)}

Hodge, P., Hellwich, K., et al.

Pure and Applied Chemistry, 2020

Volume 92(5), 797-813

https://doi.org/10.1515/pac-2018-0602

It is the goal of the IUPAC Polymer Division and the IUPAC Division of Chemical Nomenclature and Structure Representation to improve communication between polymer scientists and chemists and scientists in general by recommending unambiguous, standardized, and universally understood names and structure representations of polymers. The concise guide to polymer nomenclature is based on official IUPAC recommendations. In the online version of the report, the main points carry hyperlinks to the source documents and IUPAC color books, i.e. the Gold Book, Purple Book (polymers), Blue Book (organic chemistry), etc.

The report includes sections on source-based nomenclature, on structure-based nomenclature, on traditional names of polymers, on abbreviations, and on polymer class names.

https://iupac.org/project/2008-020-1-400

\title{
IUPAC Provisional Recommendations
}

Provisional Recommendations are preliminary drafts of IUPAC recommendations. These drafts encompass topics including terminology, nomenclature, and symbols. Following approval, the final recommendations are published in IUPAC's journal Pure and Applied Chemistry (PAC) or in IUPAC books. During the commentary period for Provisional Recommendations, interested parties are encouraged to suggest revisions to the recommendation's author.

Structure-Based Nomenclature for Irregular Linear, Star, Comb and Brush Polymers

Comments by 31 December 2020

Corresponding Author: Jiazhong Chen < Jiazhong.

Chen@dupont.com>

https://iupac.org/project/2019-036-1-800

\section{Metrological and quality concepts in analytical chemistry}

Comments by 31 December 2020

Corresponding Author: D. Brynn Hibbert <b.hibbert@ unsw.edu.au>

https://iupac.org/project/2012-007-1-500 